\title{
High-flow nasal cannula for respiratory evaluation of surfactant protein $\mathrm{C}$ dysfunction
}

\author{
Masao Nogami ${ }^{1}$, Soichiro Wada ${ }^{1}$, Shuji Sai ${ }^{1}$, Taiji Nakashima ${ }^{2}$, Tetuo Onda ${ }^{3}$, and K. Cho ${ }^{3}$ \\ ${ }^{1}$ Teine Keijinkai Hospital \\ ${ }^{2}$ Japan Community Health Care Organisation Hokkaido Hospital \\ ${ }^{3}$ Hokkaido University Hospital
}

December 16, 2020

\begin{abstract}
Surfactant protein C gene (SFTPC) variations may cause hereditary interstitial lung disease (HILD), a disease with variable prognosis. The evaluation of treatment responsiveness of HILD with SFTPC variation remains unclear. We present the case of a child with HILD whose respiratory condition was appropriately evaluated using a high-flow nasal cannula (HFNC). A 12month-old boy with SFTPC variation developed progressive respiratory failure. Although he was once considered a candidate for lung transplantation, his respiratory condition was improved by hydroxychloroquine treatment, which prevented further therapy. Compared to a conventional nasal cannula, which could not deliver a stable oxygen concentration, HFNC allowed us to evaluate the improvement of oxygenation more accurately.
\end{abstract}

\section{Title of Manuscript:}

High-flow nasal cannula for respiratory evaluation of surfactant protein $\mathbf{C}$ dysfunction

Authors: Masao Nogami MD ${ }^{1}$, Soichiro Wada $\mathrm{MD}^{1}$, Shuji Sai MD $\mathrm{PhD}^{1}$, Taiji Nakashima MD $\mathrm{PhD}^{2}$, Tetsuo Onda $\mathrm{MD}^{3}$, Kazutoshi Cho MD $\mathrm{PhD}^{3}$

\section{Affiliation s:}

${ }^{1}$ Department of pediatrics, Teine Keijinkai Hospital, Sapporo, Japan

${ }^{2}$ Department of pediatrics, Japan Community Health Care Organization Hokkaido Hospital, Sapporo, Japan

${ }^{3}$ Maternity and Perinatal Care Center, Hokkaido University Hospital, Sapporo, Japan

Keywords: high-flow nasal cannula, surfactant protein C, hereditary interstitial lung disease, hydroxychloroquine

\section{Corresponding author:}

Masao Nogami MD, Department of Pediatrics, Teine Keijinkai Hospital

1-12 Maeda, Teine-ku, Sapporo 006-8555, Japan

Email: n.masao.cab7@gmail.com

Tel: +81 11681 8111, Fax: +81 116852196

Funding Source: No funding was received for this report.

Financial Disclosure : The authors have no financial relationships relevant to this article to disclose. 
Potential Conflicts of Interest : The authors have no potential conflicts of interest to disclose.

Short title: HFNC for respiratory evaluation of SP-C dysfunction

\section{Introduction}

Hereditary interstitial lung disease (HILD) associated with surfactant protein C gene $(S F T P C$ ) variation exhibits various phenotypes, including asymptomatic adults or severe respiratory failure in infancy. ${ }^{1}$ Although several case reports have shown success with hydroxychloroquine (HCQ) treatment, other factors that can predict treatment responsiveness or disease prognosis remain largely unknown. Improvement of oxygenation was often used as a clinical parameter, ${ }^{2}$ but it is difficult to evaluate the oxygenation under specific oxygen concentrations when using conventional oxygen therapy in young children. ${ }^{3}$ Especially for patients with HILD, the oxygen concentration via a nasal cannula can vary due to respiratory distress. Here, we report that a high-flow nasal cannula (HFNC) was useful for clinical evaluation in the case of HILD with SFTPC variation.

\section{Case}

A 12-month-old Japanese boy with interstitial lung disease was transferred to our hospital due to progressive respiratory failure despite receiving systemic corticosteroids, including methylprednisolone pulse therapy. He was born at full term from nonconsanguineous parents. His past medical history was only significant for mild motor developmental delay. Family history revealed that his father was undergoing further investigation of a chest X-ray abnormality. On admission, vital signs were remarkable for tachypnea and hypoxia. The respiratory rate was $50 / \mathrm{min}$. $\mathrm{SpO}_{2}$ was variable between $90-95 \%$ with oxygen supplementation at $2 \mathrm{~L} / \mathrm{min}$ via a nasal cannula. His height and weight were $74.0 \mathrm{~cm}$ (50th percentile) and $8.1 \mathrm{~kg}$ (10th percentile), respectively. He showed moderate intercostal retraction. Respiratory distress worsened even after the second methylprednisolone pulse therapy. A week after admission, his respiratory rate increased to $70 / \mathrm{min}$, and his oxygen requirements were up to $3 \mathrm{~L} /$ min to maintain $\mathrm{SpO}_{2}>90 \%$. HCQ was administered orally to improve his respiratory condition. In the eventual case of unresponsiveness, we discussed lung transplantation with his parents and consulted a transplant surgeon. Because $\mathrm{SpO}_{2}$ with the conventional nasal cannula varied greatly depending on his activity, such as sleeping or moving, we used HFNC (Optiflow junior, OPT316, Fisher \& Paykel Healthcare Co. Ltd., Auckland, New Zealand) with an inspiratory oxygen fraction of 0.60 and flow rate of $15 \mathrm{~L} / \mathrm{min}$ to accurately monitor his oxygenation. The inspiratory oxygen fraction was adjusted to maintain $\mathrm{SpO}_{2}$ [?]95\%. Because the $\mathrm{SpO}_{2}$ variation dramatically decreased with HFNC, we were able to evaluate his oxygenation more accurately. After two weeks of HCQ administration, his oxygen requirements gradually decreased. At that time, SFTPC sequencing revealed a known pathogenic variation (c. $218 \mathrm{~T}>\mathrm{C}$, p. Ile73Thr). His respiratory distress improved so that we could change the HFNC to a conventional nasal cannula after five weeks of HCQ treatment. Corticosteroid therapy was carefully tapered because he temporarily experienced adrenal insufficiency. Four months after admission, he was discharged with home oxygen therapy and treatment with HCQ, hydrocortisone, and azithromycin. The biochemical parameters of lung injury, such as lactate dehydrogenase (LDH) and Krebs von den Lungen6 (KL-6), fluctuated during the course of inpatient treatment. The decrease in these parameters did not parallel with his clinical improvement (Figure 1). Radiological findings also improved several months after the clinical improvement.

\section{Discussion}

This report highlights two significant aspects. First, HFNC enabled us to accurately evaluate his oxygenation as a parameter of treatment effectiveness. When his diagnosis was made, it was difficult to predict the treatment responsiveness and prognosis as previous reports. ${ }^{1,2}$ Because we would have needed to consider lung transplantation if the treatment had not worked effectively, a precise assessment of the treatment response was crucial for his management. Previous studies have reported that the oxygenation improvement and nocturnal respiratory rate were adequate indicators of treatment responsiveness, although few reports described the details of these parameters. ${ }^{2}$ The guidelines for idiopathic pulmonary fibrosis in adults recommend pulmonary function tests, 6-min working tests, and improvement of CT findings for treatment 
evaluation. ${ }^{4}$ However, these investigations are challenging in infants and young children. Repeated radiological studies involve radiation exposure and the necessity for sedation in young children. In our case, we used oxygen concentration delivered by HFNC adjusted to maintain $\mathrm{SpO}_{2}$ [?]95\% to evaluate treatment responsiveness. It has been reported that oxygen concentration delivered to the patient through conventional nasal cannula varies depending on the patient's respiratory condition. ${ }^{3}$ HILD patients are supposed to be susceptible to tachypnea and hypoxia due to decreased lung compliance and diffusion impairment, compared to patients with infectious lung diseases. Therefore, an accurate oxygenation evaluation may be difficult, especially in HILD patients receiving conventional oxygen therapy. Because HFNC can provide a specific oxygen concentration, independent of the patient's respiratory conditions, it was possible to properly assess oxygen saturation even in the presence of severe respiratory distress. ${ }^{3}$ In our case, this precise assessment stratified whether he needed further therapy such as lung transplantation.

Second, the biochemical parameters of lung injury did not reflect his actual clinical conditions. Although KL-6 elevation has been reported to be helpful in the pediatric interstitial lung disease diagnosis, ${ }^{5}$ whether the biochemical parameters can reflect respiratory improvements in HILD patients remains unknown. In our case, the decrease in LDH and KL-6 levels was only evident after discharge. We presumed that the complex pathophysiology of surfactant dysfunction might cause this delay. The abnormal pro-surfactant protein $\mathrm{C}$ translated from mutant $S F T P C$ was thought to induce endoplasmic reticulum stress and inhibit the function of a normally-matured surfactant protein C. ${ }^{1}$ Consequently, pulmonary inflammation and pulmonary surfactant dysfunction might cause respiratory insufficiency. ${ }^{1}$ The decrease in biochemical parameters did not match the improvement of respiratory condition, as these parameters only reflected the resolution of lung inflammation. We speculated that the recovery of surfactant function occurred initially, followed by the resolution of lung inflammation. Although it was not possible to explain this phenomenon precisely, physiological parameters seemed to be more helpful than biochemical parameters to evaluate the treatment responsiveness, at least in our case.

The concrete evidence of HFNC usefulness as a respiratory support for HILD remains to be investigated. Nevertheless, our findings may be useful to evaluate the respiratory condition of HILD with SFTPC variations.

\section{Reference}

1. Wert SE, Whitsett JA, Nogee LM. Genetic disorders of surfactant dysfunction. Pediatr Dev Pathol 2009;12(4):253-74.

2. Klay D, Hoffman TW, Harmsze AM, Grutters JC, van Moorsel CHM. Systematic review of drug effects in humans and models with surfactant-processing disease. Eur Respir Rev 2018;27(149): 170135.

3. Walsh BK, Smallwood CD. Pediatric oxygen therapy: a review and update. Respir Care 2017;62(6):64561.

4. Raghu G, Collard HR, Egan JJ, Martinez FJ, Behr J, Brown KK, Colby TV, Cordier JF, Flaherty KR, Lasky JA, et al. An official ATS/ERS/JRS/ALAT statement: idiopathic pulmonary fibrosis: evidence-based guidelines for diagnosis and management. Am J Respir Crit Care Med 2011;183(6):788-824.

5. Al-Salmi QA, Walter JN, Colasurdo GN, Sockrider MM, Smith EO, Takahashi H, Fan LL. Serum KL-6 and surfactant proteins A and D in pediatric interstitial lung disease. Chest 2005;127(1):403-7.

\section{Hosted file}

Figure Legends.pdf available at https://authorea.com/users/383358/articles/499236-high-flownasal-cannula-for-respiratory-evaluation-of-surfactant-protein-c-dysfunction 


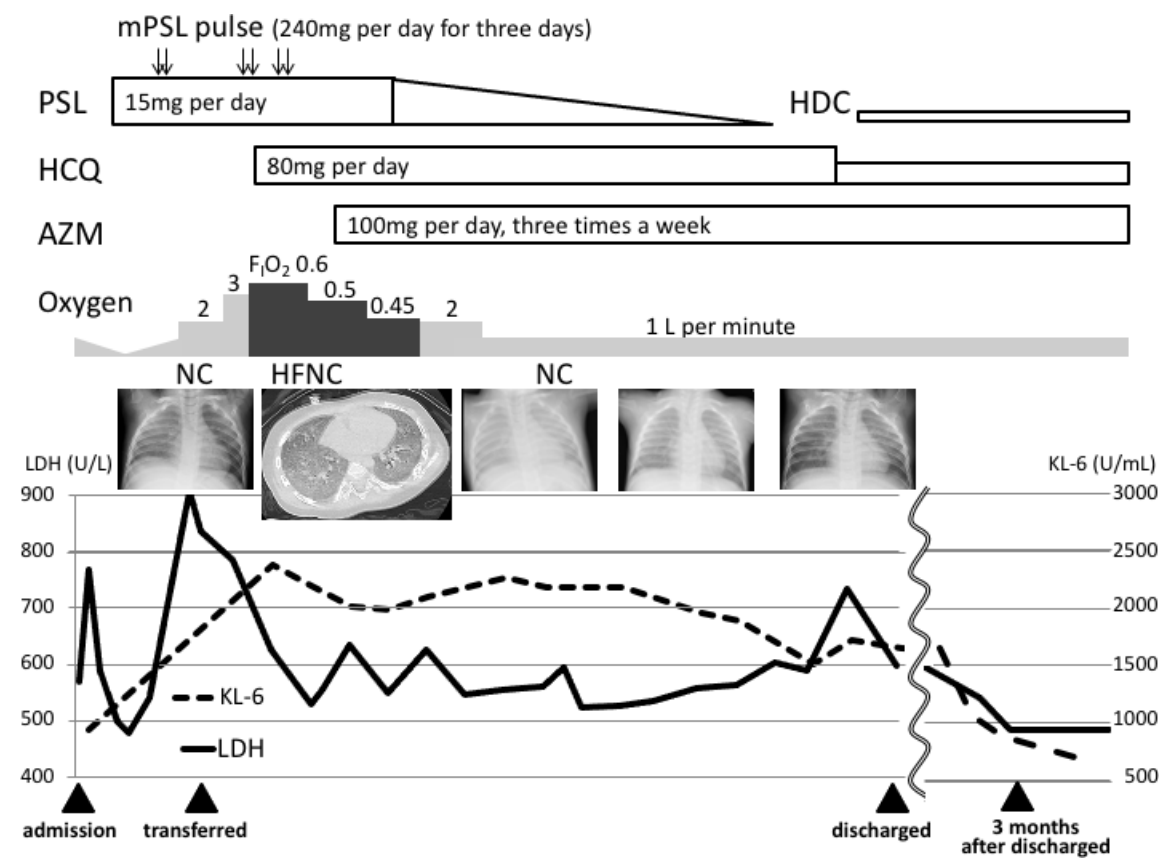

